\title{
Mutation analysis of key genes in RAS/RAF and PI3K/PTEN pathways in Chinese patients with hepatocellular carcinoma
}

\author{
WENMIN HOU ${ }^{1,2^{*}}$, JIBIN LIU $^{2,3^{*}},{\text { PEIZHAN } \mathrm{CHEN}^{2}, \text { HUI WANG }^{2}, \text { BANG-CE YE }^{1} \text { and FULIN QIANG }}^{3}$ \\ ${ }^{1}$ Laboratory of Biosystems and Microanalysis, State Key Laboratory of Bioreactor Engineering, \\ East China University of Science and Technology, Shanghai 200237; ${ }^{2}$ Key Laboratory of Food Safety Research, \\ Institute for Nutritional Sciences, Shanghai Institutes for Biological Sciences, Chinese Academy of Sciences, \\ University of Chinese Academy of Sciences, Shanghai 200031; ${ }^{3}$ Tumor Institute, \\ Nantong Tumor Hospital, Nantong, Jiangsu 226000, P.R. China
}

Received November 30, 2013; Accepted April 4, 2014

DOI: $10.3892 / \mathrm{ol} .2014 .2253$

\begin{abstract}
The RAS/RAF and PI3K/PTEN signaling pathways play central roles in hepatocarcinogenesis. KRAS, NRAS, HRAS, $B R A F, P I K 3 C A, P I K 3 R I$ and PTEN are key cancer-related genes in the RAS/RAF and PI3K/PTEN signaling pathways. Genetic alterations in these genes often lead to the dysregulation of the two cascades. Little is known regarding the frequency of hotspot mutations in these critical components among Chinese patients with hepatocellular carcinoma (HCC). In the current study, 57 somatic hotspot mutations in 36 HCCs samples collected from Chinese patients using direct DNA sequencing method were examined. Two cases of KRAS somatic mutations (KRAS codon 61; Gln to His) were identified among $36 \mathrm{HCCs}$ (5.6\%). However, no mutations were found in the NRAS, HRAS, $B R A F, P I K 3 C A, P I K 3 R 1$ and PTEN genes. These findings indicated that point mutations in the KRAS gene, but not mutations in NRAS, HRAS, BRAF, PIK3CA, PIK3RI and PTEN genes, at a somatic level contribute to the abnormal activation of the RAS/RAF and PI3K/PTEN pathways in HCC.
\end{abstract}

\section{Introduction}

Hepatocellular carcinoma (HCC) is one of the most common malignancies worldwide, accounting for $>740,000$ new cases

Correspondence to: Professor Fulin Qiang, Tumor Institute, Nantong Tumor Hospital, 30 Tongyang Beilu, Nantong, Jiangsu 226000, P.R. China

E-mail: nantongflqiang@gmail.com

Professor Bang-Ce Ye, Laboratory of Biosystems and Microanalysis, State Key Laboratory of Bioreactor Engineering, East China University of Science and Technology, 120 Meilong Road, Shanghai 200237, P.R. China

E-mail: bcye@ecust.edu.cn

*Contributed equally

Key words: hepatocellular carcinoma, mutation analysis, KRAS, cancer-related genes and 690,000 mortalities per year (1). Half of these new cases and mortalities were estimated to occur in China. The high rates of $\mathrm{HCC}$ in China are largely due to the prevalence of chronic hepatitis B virus (HBV) infection (2). The RAS/RAF and PI3K/PTEN signaling pathways play central roles in hepatocarcinogenesis (3). The aberrant activation of the RAS/RAF and PI3K/PTEN signaling pathways is associated with poor prognosis in cancer patients $(4,5)$. HBV also utilizes the pathways for the control of hepatocyte survival and viral replication $(6,7)$. Mutations of key components (such as RAS, RAF, PIK3CA, PIK3R1 and PTEN) in the RAS/RAF and PI3K/PTEN pathways lead to the dysregulation of the two cascades (8). The RAS family comprises three members: KRAS, NRAS and HRAS. Somatic mutations in the $R A S$ family are common in numerous human cancer types, including pancreatic, thyroid, colorectal, liver, kidney and lung (9). BRAF is the most frequently mutated gene in the $R A F$ family, and the $B R A F$ mutation has been reported in $61 \%$ of melanoma, $53 \%$ of papillary thyroid cancer and $11.5 \%$ of colorectal cancer patients (10-12). The PI3K gene comprises $P I K 3 C A$, which encodes the catalytically active p110 $\alpha$ subunit, and $P I K 3 R 1$, encoding the p $85 \alpha$ regulatory subunit (13). PIK3CA is mutated in numerous tumor types, with the frequency ranging from 4 to $32 \%$ in breast, colorectal, endometrial, brain, gastric and lung cancer (14-17). PIK3RI mutations were identified in $43 \%$ of endometrial cancer, $4 \%$ of ovarian cancer and $2 \%$ of colon cancer (18-19). PTEN acts as a negative regulator of the PI3K pathway and PTEN mutations lead to a reduction of its phosphatase activity (20). Mutations of the PTEN gene are associated with a wide variety of human tumors (21).

Inhibitors targeting the RAS/RAF and PI3K/PTEN pathways have been developed and the clinical responses of patients were observed to differ according to the genetic alterations of the critical components of the two cascades (22). However, few data are available regarding the prevalence of KRAS, NRAS, HRAS, BRAF, PIK3CA, PIK3R1 and PTEN mutations in Chinese patients with HCC. In the present study, we conducted mutational analysis of 57 somatic hotspot mutations in KRAS, NRAS, HRAS, BRAF, PIK3CA, PIK3RI and $P T E N$ in 36 Chinese patients with HCC. 


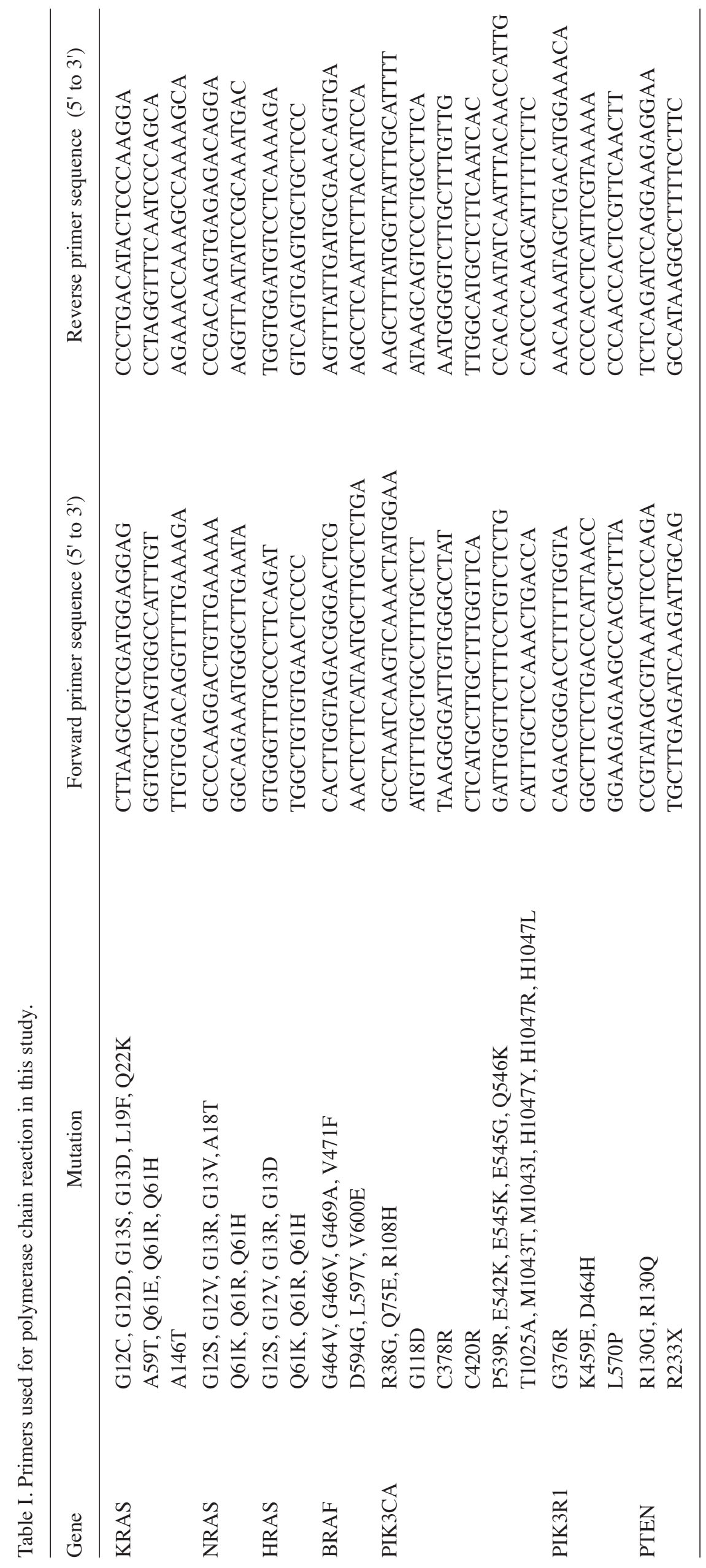




\section{Materials and methods}

Patients and tissue samples. Thirty-six patients with HCC undergoing surgery at Nantong Tumor Hospital (Nantong, China) between 2009 and 2011 were enrolled in this study. Tumor samples and adjacent normal liver tissues from the corresponding patients were fixed with $10 \%$ formalin, embedded in paraffin and stained with hematoxylin and eosin (H\&E). Tumor staging was performed according to the Barcelona Clinic Liver Cancer (BCLC) staging classification (23). This study was approved by the Ethics Committee of Nantong Tumor Hospital. Written informed consent was obtained from each patient prior to sample collection.

Genomic DNA extraction. Tumor areas and non-tumorous tissue areas were identified on H\&E-stained slides. Genomic DNA was extracted from formalin-fixed paraffin-embedded tissues of HCC with the QIAamp DNA FFPE Tissue kit (Qiagen GmbH, Hilden, Germany) according to the manufacturer's instructions. Briefly, samples were placed into Eppendorf tubes and the paraffin was removed. Next, the tubes were incubated with proteinase $\mathrm{K}$ (Qiagen $\mathrm{GmbH}$ ) at $56^{\circ} \mathrm{C}$ for $1 \mathrm{~h}$. Following proteinase $\mathrm{K}$ digestion, the samples were incubated at $90^{\circ} \mathrm{C}$ for $1 \mathrm{~h}$ and DNA was extracted using QIAamp MinElute columns (Qiagen $\mathrm{GmbH}$ ).

Mutation analysis. Polymerase chain reaction (PCR) was performed to amplify the gene fragments including the hotspot mutations shown in Table I. The selection of the hotspots was based on the prevalence of mutations in cancers identified in the COSMIC database (24). A $50 \mu \mathrm{l}$ volume of PCR was prepared using the Taq PCR Master Mix kit (Qiagen $\mathrm{GmbH}$ ), according to the manufacturer's instructions. The thermocycling was performed at $94^{\circ} \mathrm{C}$ for $3 \mathrm{~min}$; 35 cycles of $94^{\circ} \mathrm{C}$ for $30 \mathrm{sec}, 56^{\circ} \mathrm{C}$ for $30 \mathrm{sec}$ and $72^{\circ} \mathrm{C}$ for $60 \mathrm{sec}$; followed by a final $10 \mathrm{~min}$ at $72^{\circ} \mathrm{C}$. PCR products were run on $1.5 \%$ agarose gel electrophoresis and visualized with ultraviolet light to confirm sizes. DNA purification was performed with the QIAprep Gel Extraction kit (Qiagen $\mathrm{GmbH}$ ) according to the manufacturer's instructions. Briefly, the DNA fragments were excised from the agarose gel with a scalpel and placed in a colorless tube. DNA cleanup was conducted using QIAquick spin columns (Qiagen $\mathrm{GmbH}$ ). Direct DNA sequencing was performed using a Big Dye Terminator (v3.1) kit (Applied Biosystems, Foster City, CA, USA). The sequencing products were run on an Applied Biosystems 3130XL Genetic Analyzer (Applied Biosystems). DNA sequencing results were analyzed using Chromas software (Technelysium, Brisbane, Queensland, Australia). The primers used for the PCR are listed in Table I.

\section{Results}

Clinicopathological characteristics. Of 36 patients with HCC, the median age was 54 years (range, 40-77 years), including 33 males and three females. The majority of the cases had HCC associated with HBV infection (34/36; 94.4\%). All patients were negative in hepatitis $C$ virus infection. The concentrations of serum AFP of 16 patients $(16 / 36 ; 44.4 \%)$ were higher than $400 \mathrm{ng} / \mathrm{ml}$. The BCLC staging classification was used to
Table II. Clinical characteristics of hepatocellular carcinoma patients.

\begin{tabular}{lc}
\hline Characteristic & Value \\
\hline Age, years & \\
Median (range) & $54(40-77)$ \\
Gender, $\mathrm{n}(\%)$ & \\
Male & $33(91.7)$ \\
Female & $3(8.3)$ \\
Etiology, $\mathrm{n}(\%)$ & \\
$\mathrm{HBV}(+)$ & $34(94.4)$ \\
$\mathrm{HBV}(-)$ & $2(5.6)$ \\
AFP, $\mathrm{n}(\%)$ & \\
$>400 \mathrm{ng} / \mathrm{ml}$ & $16(44.4)$ \\
$\leq 400 \mathrm{ng} / \mathrm{ml}$ & $20(55.6)$ \\
Stage, $\mathrm{n}(\%)$ & \\
0 & $2(5.6)$ \\
$\mathrm{A}$ & $25(69.4)$ \\
$\mathrm{B}$ & $8(22.2)$ \\
$\mathrm{C}$ & $1(2.8)$ \\
$\mathrm{D}$ & $0(0.0)$ \\
\hline
\end{tabular}

classify the cancer staging (23). There were 2, 25, 8, 1 and 0 cases of stages 0 to D, respectively (Table II).

Mutationanalysis of keygenes in the RAS/RAF andPI3K/PTEN pathways. We analyzed hotspot-containing gene fragments of key genes in RAS/RAF and PI3K/PTEN pathways using PCR amplification followed by direct sequencing. The hotspots were listed in Table I. In all, two samples (Sample \#13 and \#35) had point mutations in codon $61(\mathrm{Q} 61 \mathrm{H})$ of the KRAS gene and the mutation rate was $5.6 \%$ (Fig. 1). In the two cases, codon 61 was altered from CAA, coding for Gln, to CAC, coding for His. To confirm the two mutations occurred at the somatic level, we tested codon 61 mutation status in non-tumorous tissues from the two patients. The results showed that codon 61 was wild-type in normal tissues from Sample \#13 and \#35 (Fig. 1). The two patients harboring KRAS mutation were male. Patient no. 13 was 49 years old, had HBV infection and stage A HCC, and an AFP level of $3.4 \mathrm{ng} / \mathrm{ml}$. Patient no. 35 was 69 years old, had stage B HCC and was negative for HBV infection, with an AFP level of $1.95 \mathrm{ng} / \mathrm{ml}$. No other mutations in the HRAS, NRAS, BRAF, PIK3CA, PIK3RI and PTEN genes were identified.

\section{Discussion}

Targeting the RAS/RAF and PI3K/PTEN pathways are novel therapeutic strategies that may be exploited for the treatment of HCC (8). As the RAF-kinase inhibitor sorafenib has been demonstrated to be effective in the treatment of HCC, BRAF mutations have become a favored target in HCC treatment recently (25). However, the somatic mutation prevalence and distribution of the key genes in the two pathways remain largely unknown in Chinese patients with HCC. Therefore, the present 
Table III. Reported point mutations in codons 12, 13, and 61 of KRAS in hepatocellular carcinomas.

\begin{tabular}{|c|c|c|c|c|c|c|}
\hline Author (ref) & Population & No. of patients & Codon 12 & Codon 13 & Codon 61 & Frequency $(\%)$ \\
\hline Zuo et al (36) & Chinese & 64 & 2 & 1 & NA & 4.7 \\
\hline Huang et al (31) & Chinese & 10 & 0 & 0 & 0 & 0.0 \\
\hline Taketomi et al (33) & Japanese & 61 & 0 & 1 & 0 & 1.6 \\
\hline Tsuda et al (43) & Japanese & 30 & 1 & 0 & 0 & 3.3 \\
\hline Taniguchi et al (44) & Japanese & 15 & 0 & 0 & NA & 0.0 \\
\hline Fujimoto et al (29) & Japanese & 27 & 0 & 0 & 0 & 0.0 \\
\hline Tada et al (45) & Japanese & 12 & 0 & 0 & 0 & 0.0 \\
\hline Bose et al (46) & Indian & 30 & 2 & 0 & 0 & 6.7 \\
\hline Tannapfel et al (27) & German & 25 & 0 & 0 & NA & 0.0 \\
\hline Weihrauch et al (47) & German & 20 & 3 & 0 & NA & 15.0 \\
\hline Challen et al (32) & British & 19 & 0 & NA & 1 & 5.3 \\
\hline Guichard et al (30) & French & 149 & 1 & 0 & 1 & 1.3 \\
\hline Colombino et al (37) & Italian & 65 & 1 & 0 & 0 & 1.5 \\
\hline
\end{tabular}

NA, not available.

A

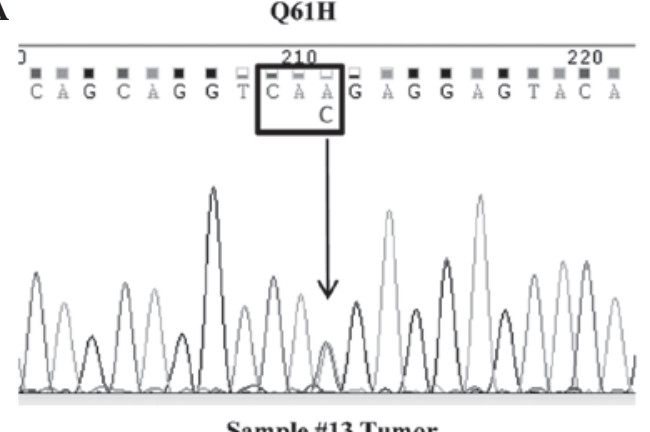

Sample \#13 Tumor

B

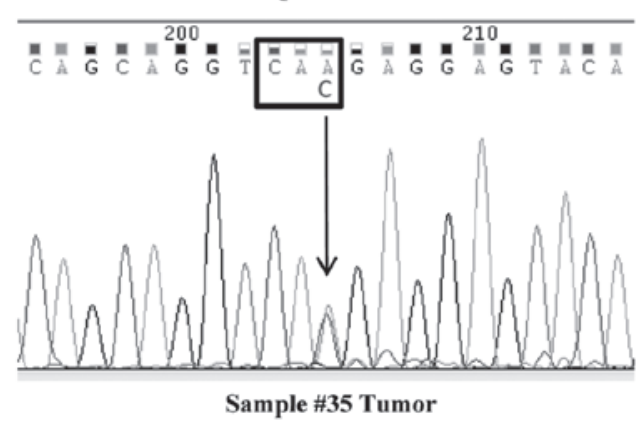

Q61 Wild Type

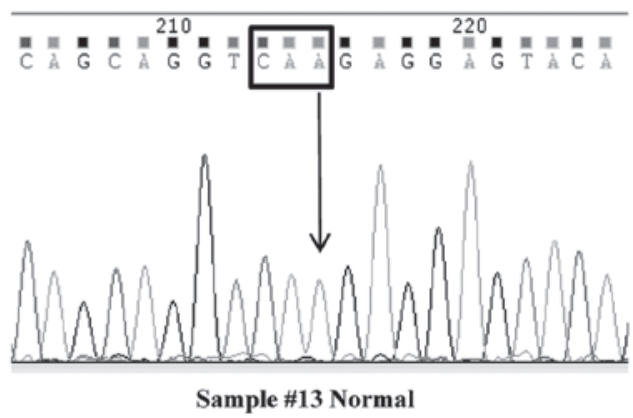

Q61 Wild Type

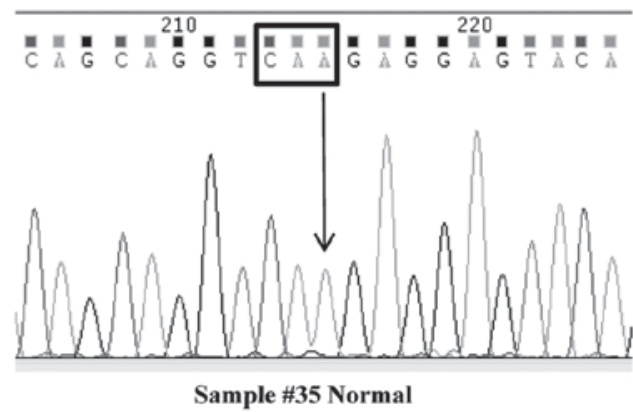

Figure 1. Sequence analysis of KRAS gene mutation in codon 61 in two hepatocellular carcinoma cases. Sequence chromatograms of codon 61 in tumor and normal tissues from (A) Sample \#13 and (B) Sample \#35.

study set out to examine the frequency of hotspot mutations of the KRAS, NRAS, HRAS, BRAF, PIK3CA, PIK3RI and PTEN genes in 36 human HCC tissues from Chinese patients. Only $K R A S$ somatic mutations were identified, with a mutation rate of $5.6 \%$.

The incidence of KRAS mutations has been found in $80 \%$ of advanced pancreatic cancer (26), $45 \%$ of cholangiocarcinoma (27) and $32 \%$ of colorectal cancer (28) patients. COSMIC database has shown that mutations in codons 12, 13 and 61 of the KRAS gene are known hotspots in various types of cancer. The frequency and distribution of KRAS mutation in HCC from several previous studies are summarized in Table III. The majority of these studies have shown that KRAS gene mutations occur infrequently $(<10 \%)$ in HCC. The codon 12 accounts for the majority of KRAS mutations detected $(\sim 70 \%)$, whereas mutations affecting codon 13 and codon 61 account for the remaining 
$30 \%$. One third of the twelve studies did not evaluate the KRAS codon 61 mutation status, which may cause bias in the distribution of the KRAS mutation. Three whole exome sequencing studies conducted mutational screening in all KRAS exons and found that the mutations were clustered in the hotspots (29-31). In the current study, mutations were detected in codons 12 , 13 and 61 of the KRAS gene, and two out of $36(5.6 \%) \mathrm{HCCs}$ harbored KRAS mutations in codon 61. Therefore, KRAS gene mutations may participate in hepatocellular carcinogenesis.

The present study also investigated the hotspot mutations in NRAS and HRAS, but found no mutation in the two genes. Few studies have focused on the mutation incidence of these two RAS family members in Chinese patients with HCC. A whole exome sequencing study identified no mutation in these two genes in a Chinese population (31). Challen et al found that the frequency of NRAS mutations was $15.8 \%$, but did not identify HRAS mutations, in British patients with HCC (32). Taketomi et al reported neither NRAS nor HRAS mutations were detected in Japanese HCC cases (33). Thus, the mutational activation of NRAS and HRAS genes is an uncommon event in the pathogenesis of HCCs.

$B R A F$ mutations can abnormally activate downstream signaling pathways in HCC and act as indicator of cetuximab resistance in patients with colon cancer $(34,35)$. BRAF mutations are believed to be rare in HCCs. Previously, no $B R A F$ mutations were identified in German and Chinese populations $(27,36)$. However, Colombino et al detected that the $B R A F$ gene was highly mutated in $\sim 23 \%$ of Italian HCC cases (37). In the current series, no $B R A F$ mutations were observed, indicating that $B R A F$ mutation does not play a major role in abnormal activation of RAS/RAF signaling pathway.

PIK3CA, PIK3R 1 and PTEN are key genes in the PI3K/PTEN pathway (8). In the current study, it was found that mutations were absent in the three genes. Previously, PIK3CA was observed to be frequently mutated in Korean and Italian patients with HCC, with mutation rates of 35.6 and $28 \%$, respectively $(15,37)$. However, Tanaka et al did not identify PIK3CA mutations in Japanese patients with HCC, and Riener et al reported that the PIK3CA mutation incidence was $2 \%$ in Swiss patients with HCC $(38,39)$. In two studies in Chinese patients with $\mathrm{HCC}$, the mutation rates were 1.6 and $1.1 \%(36,40)$, which were similar to those of the present study. The conflicting data may be due to the different genetic backgrounds of the populations, $\mathrm{HBV}$ infection status and smaller sample size in the current study. PIK3RI mutation has been found to occur infrequently in numerous cancer types, including ovarian and colon cancer (19), and the present study showed a low frequency of alteration of PIK $3 R 1$ in HCC. Inactivation of PTEN in HCC may be largely due to frequent loss of heterozygosity of the PTEN allele; the frequency was identified to be $\leq 44.4 \%$ (41). Wang et al investigated PTEN mutations in exons 5 and 8 , but failed to detect any (42), which was in agreement with the results of the present study. Mutations in the PIK3CA, PIK3RI and PTEN genes rarely occur in HCC, suggesting that somatic point mutations of these three genes may not play an important role in HCC in the Chinese population. However, further research is necessary to confirm these results in larger sample size.

In summary, the present study investigated the prevalence of KRAS, NRAS, HRAS, BRAF, PIK3CA, PIK3RI and
PTEN mutations in 57 hotspot mutations. Two cases of KRAS mutation were identified among $36 \mathrm{HCC}$ cases. The findings indicated that point mutations in the KRAS gene, but not mutations in the NRAS, HRAS, BRAF, PIK3CA, PIK3RI and PTEN genes, at the somatic level contribute to the abnormal activation of the RAS/RAF and PI3K/PTEN pathways in HCC. Considering the low frequency of key genes in the RAS/RAF and PI3K/PTEN signaling pathways, other mechanisms to activate the RAS/RAF and PI3K/PTEN pathways, such as gene amplification, deletion, and aberrant methylation, may be involved in the development and progression of HCC.

\section{Acknowledgements}

This study was supported by grants from the Key Research Program of the Chinese Academy of Sciences (grant no. KSZD-EW-Z-019), the National Nature Science Foundation (grant nos. 31101261, 81302507 and 81302809), the Ministry of Science and Technology of China (grant no. 2014AA020524), the Shanghai Institutes for Biological Sciences, Chinese Academy of Sciences (grant nos. 2012KIP308 and 2012KIP515) and the Food Safety Research Center and Key Laboratory of Food Safety Research of INS, SIBS, CAS. Dr. Peizhan Chen was partially supported by the SA-SIBS scholarship program.

\section{References}

1. Jemal A, Bray F, Center MM, Ferlay J, Ward E and Forman D: Global cancer statistics. CA Cancer J Clin 61: 69-90, 2011.

2. McGlynn KA and London WT: The global epidemiology of hepatocellular carcinoma: present and future. Clin Liver Dis 15: 223-243, vii-x, 2011.

3. Owonikoko TK and Khuri FR: Targeting the PI3K/AKT/mTOR pathway: biomarkers of success and tribulation. Am Soc Clin Oncol Educ Book: 2013. doi: 10.1200/EdBook_AM.2013.33.e395.

4. McCubrey JA, Steelman LS, Abrams SL, et al: Targeting survival cascades induced by activation of Ras/Raf/MEK/ERK, PI3K/PTEN/Akt/mTOR and Jak/STAT pathways for effective leukemia therapy. Leukemia 22: 708-722, 2008.

5. Downward J: Targeting RAS signalling pathways in cancer therapy. Nat Rev Cancer 3: 11-22, 2003.

6. Zheng Y, Li J, Johnson DL and Ou JH: Regulation of hepatitis B virus replication by the ras-mitogen-activated protein kinase signaling pathway. J Virol 77: 7707-7712, 2003.

7. Liu H, Xu J, Zhou L, et al: Hepatitis B virus large surface antigen promotes liver carcinogenesis by activating the Src/PI3K/Akt pathway. Cancer Res 71: 7547-7557, 2011.

8. Steelman LS, Chappell WH, Abrams SL, et al: Roles of the $\mathrm{Raf} / \mathrm{MEK} / \mathrm{ERK}$ and PI3K/PTEN/Akt/mTOR pathways in controlling growth and sensitivity to therapy-implications for cancer and aging. Aging (Albany NY) 3: 192-222, 2011.

9. Fernández-Medarde A and Santos E: Ras in cancer and developmental diseases. Genes Cancer 2: 344-358, 2011.

10. Fransén K, Klintenäs M, Osterström A, Dimberg J, Monstein HJ and Söderkvist P: Mutation analysis of the BRAF, ARAF and RAF-1 genes in human colorectal adenocarcinomas. Carcinogenesis 25: 527-533, 2004.

11. Libra M, Malaponte G, Navolanic PM, et al: Analysis of BRAF mutation in primary and metastatic melanoma. Cell Cycle 4: 1382-1384, 2005.

12. Fukushima T, Suzuki S, Mashiko M, et al: BRAF mutations in papillary carcinomas of the thyroid. Oncogene 22: 6455-6457, 2003.

13. Roymans D and Slegers H: Phosphatidylinositol 3-kinases in tumor progression. Eur J Biochem 268: 487-498, 2001.

14. Samuels Y, Wang Z, Bardelli A, et al: High frequency of mutations of the PIK3CA gene in human cancers. Science 304: $554,2004$. 
15. Lee JW, Soung YH, Kim SY, et al: PIK3CA gene is frequently mutated in breast carcinomas and hepatocellular carcinomas. Oncogene 24: 1477-1480, 2005.

16. Shayesteh L, Lu Y, Kuo WL, et al: PIK3CA is implicated as an oncogene in ovarian cancer. Nat Genet 21: 99-102, 1999.

17. Ligresti G, Militello L, Steelman LS, et al: PIK3CA mutations in human solid tumors: role in sensitivity to various therapeutic approaches. Cell Cycle 8: 1352-1358, 2009.

18. Urick ME, Rudd ML, Godwin AK, Sgroi D, Merino M and Bell DW: PIK3R1 (p85alpha) is somatically mutated at high frequency in primary endometrial cancer. Cancer Res 71: 4061-4067, 2011.

19. Philp AJ, Campbell IG, Leet C, et al: The phosphatidylinositol 3'-kinase p85alpha gene is an oncogene in human ovarian and colon tumors. Cancer Res 61: 7426-7429, 2001.

20. Stambolic V, Suzuki A, de la Pompa JL, et al: Negative regulation of PKB/Akt-dependent cell survival by the tumor suppressor PTEN. Cell 95: 29-39, 1998.

21. Tamguney T and Stokoe D: New insights into PTEN. J Cell Sci 120: 4071-4079, 2007.

22. Chappell WH, Steelman LS, Long JM, et al: Ras/Raf/MEK/ERK and PI3K/PTEN/Akt/mTOR inhibitors: rationale and importance to inhibiting these pathways in human health. Oncotarget 2: 135-164, 2011.

23. Llovet JM, Bru C and Bruix J: Prognosis of hepatocellular carcinoma: the BCLC staging classification. Semin Liver Dis 19: 329-338, 1999.

24. Forbes SA, Bindal N, Bamford S, et al: COSMIC: mining complete cancer genomes in the Catalogue of Somatic Mutations in Cancer. Nucleic Acids Res 39: D945-D950, 2011

25. Zhu AX: Development of sorafenib and other molecularly targeted agents in hepatocellular carcinoma. Cancer 112: 250-259, 2008

26. De La O JP and Murtaugh LC: Notch and Kras in pancreatic cancer: at the crossroads of mutation, differentiation and signaling. Cell Cycle 8: 1860-1864, 2009.

27. Tannapfel A, Sommerer F, Benicke M, et al: Mutations of the BRAF gene in cholangiocarcinoma but not in hepatocellular carcinoma. Gut 52: 706-712, 2003.

28. Balschun K, Haag J, Wenke AK, von Schonfels W, Schwarz NT and Rocken C: KRAS, NRAS, PIK3CA exon 20, and BRAF genotypes in synchronous and metachronous primary colorectal cancers diagnostic and therapeutic implications. J Mol Diagn 13: 436-445, 2011

29. Fujimoto A, Totoki Y, Abe T, et al: Whole-genome sequencing of liver cancers identifies etiological influences on mutation patterns and recurrent mutations in chromatin regulators. Nat Genet 44: 760-764, 2012

30. Guichard C, Amaddeo G, Imbeaud S, et al: Integrated analysis of somatic mutations and focal copy-number changes identifies key genes and pathways in hepatocellular carcinoma. Nat Genet 44: 694-698, 2012.

31. Huang J, Deng Q, Wang Q, et al: Exome sequencing of hepatitis B virus-associated hepatocellular carcinoma. Nat Genet 44: 1117-1121, 2012.
32. Challen C, Guo K, Collier JD, Cavanagh D and Bassendine MF: Infrequent point mutations in codons 12 and 61 of ras oncogenes in human hepatocellular carcinomas. J Hepatol 14: 342-346, 1992

33. Taketomi A, Shirabe K, Muto J, et al: A rare point mutation in the Ras oncogene in hepatocellular carcinoma. Surg Today 43: 289-292, 2013.

34. Huynh H, Nguyen TT, Chow KH, Tan PH, Soo KC and Tran E: Over-expression of the mitogen-activated protein kinase (MAPK) kinase (MEK)-MAPK in hepatocellular carcinoma: its role in tumor progression and apoptosis. BMC Gastroenterol 3: 19, 2003.

35. Di Nicolantonio F, Martini M, Molinari F, et al: Wild-type BRAF is required for response to panitumumab or cetuximab in metastatic colorectal cancer. J Clin Oncol 26: 5705-5712, 2008.

36. Zuo Q, Huang H, Shi M, et al: Multivariate analysis of several molecular markers and clinicopathological features in postoperative prognosis of hepatocellular carcinoma. Anat Rec (Hoboken) 295: 423-431, 2012.

37. Colombino M, Sperlongano P, Izzo F, et al: BRAF and PIK3CA genes are somatically mutated in hepatocellular carcinoma among patients from South Italy. Cell Death Dis 3: e259, 2012.

38. Tanaka Y, Kanai F, Tada M, et al: Absence of PIK3CA hotspot mutations in hepatocellular carcinoma in Japanese patients. Oncogene 25: 2950-2952, 2006.

39. Riener MO, Bawohl M, Clavien PA and Jochum W: Rare PIK3CA hotspot mutations in carcinomas of the biliary tract. Genes Chromosomes Cancer 47: 363-367, 2008.

40. Li X, Zhang Q, He W, et al: Low frequency of PIK3CA gene mutations in hepatocellular carcinoma in Chinese population. Pathol Oncol Res 18: 57-60, 2012.

41. Bae JJ, Rho JW, Lee TJ, et al: Loss of heterozygosity on chromosome 10q23 and mutation of the phosphatase and tensin homolog deleted from chromosome 10 tumor suppressor gene in Korean hepatocellular carcinoma patients. Oncol Rep 18: 1007-1013, 2007.

42. Wang L, Wang WL, Zhang Y, Guo SP, Zhang J and Li QL: Epigenetic and genetic alterations of PTEN in hepatocellular carcinoma. Hepatol Res 37: 389-396, 2007.

43. Tsuda H, Hirohashi S, Shimosato Y, Ino Y, Yoshida T and Terada M: Low incidence of point mutation of c-Ki-ras and N-ras oncogenes in human hepatocellular carcinoma. Jpn J Cancer Res 80: 196-199, 1989.

44. Taniguchi K, Yamada T, Sasaki Y and Kato K: Genetic and epigenetic characteristics of human multiple hepatocellular carcinoma. BMC Cancer 10: 530, 2010.

45. Tada M, Omata M and Ohto M: Analysis of ras gene mutations in human hepatic malignant tumors by polymerase chain reaction and direct sequencing. Cancer Res 50: 1121-1124, 1990.

46. Bose S, Sakhuja P, Bezawada L, et al: Hepatocellular carcinoma with persistent hepatitis B virus infection shows unusual downregulation of Ras expression and differential response to Ras mediated signaling. J Gastroenterol Hepatol 26: 135-144, 2011

47. Weihrauch M, Benicke M, Lehnert G, Wittekind C, Wrbitzky R and Tannapfel A: Frequent k- ras -2 mutations and p16(INK4A) methylation in hepatocellular carcinomas in workers exposed to vinyl chloride. Br J Cancer 84: 982-989, 2001. 